Highly Energetic Physical Processes and

Mechanisms for Emission from Astrophysical Plasmas

IAU Symposium, Vol. 195, 2000

P. C. H. Martens, S. Tsuruta, and M. A. Weber, eds.

\title{
Far-ultraviolet Emission from Supernova Remnant Shocks
}

\author{
R. Sankrit and W. P. Blair \\ Johns Hopkins University, 3400 N. Charles St., Baltimore, MD 21218, \\ U.S.A.
}

FUSE SNR Team

JHU, Princeton, GSFC, IAP, UC Berkeley

\begin{abstract}
Supernova remnant shocks produce a rich spectrum of lines in the ultraviolet. Among these are important diagnostic resonance lines of $\mathrm{O}$ VI, $\mathrm{C}$ III, and $\mathrm{N}$ III which lie in the bandpass observable with the recently launched Far Ultraviolet Spectroscopic Explorer. We present an overview of a PI program to observe several supernova remnants using this new telescope.
\end{abstract}

\section{FUSE}

The Far Ultraviolet Spectroscopic Explorer (FUSE) is a NASA Origins mission, launched on June 24,1999 . It will observe in the $905-1187 \AA$ wavelength range at a spectral resolution of about $10 \mathrm{~km} \mathrm{~s}^{-1}$. With a nominal 3 year lifetime, FUSE will be the first long duration satellite devoted to far ultraviolet spectroscopy since Copernicus. FUSE will have about 10,000 times the sensitivity of the earlier mission and will obtain spectra across the entire wavelength range simultaneously. The wavelength region covered by FUSE is rich in spectral lines that trace temperatures from $10 \mathrm{~K}$ to $10^{6} \mathrm{~K}$. These lines include molecular lines from $\mathrm{H}_{2}$ and $\mathrm{CO}$, the Lyman series of $\mathrm{HI}$, and resonance lines from many different ionization states of heavy elements.

\section{Supernova Remnants}

The far ultraviolet bandpass observable with FUSE is of particular interest for the study of supernova remnant (SNR) shocks. The O vi $\lambda \lambda 1032,1038$ resonance doublet, which traces collisionally ionized gas at about $300,000 \mathrm{~K}$, is very strong in SNR spectra. Additionally, the bandpass includes resonance lines of C III $(977 \AA)$ and N III (991 $\AA$ ) which arise in regions behind shocks where the gas has cooled below $100,000 \mathrm{~K}$. We plan to use FUSE to observe several different regions of shocked gas in the Cygnus Loop, Crab Nebula, and Vela SNRs. We present an overview of the planned observations and their scientific implications. 


\subsection{Cygnus Loop}

The Cygnus Loop is the remnant of a supernova that exploded within a cavity created by the progenitor star (see, e.g., Levenson et al. 1998). Currently, the blast wave is running into the walls of the cavity, driving shocks into the ISM. The inhomogeneity of the pre-shock medium gives rise to a range of shock properties. Thin, faint Balmer-dominated filaments trace non-radiative shock fronts where the shocked gas has not had time to cool and recombine. The bright optical filaments are regions where the pre-shock medium is relatively dense and enough material has been swept up to make the shock radiative.

We will observe a non-radiative filament and use the $\mathrm{O}$ VI line strength as a diagnostic for the shock velocity. By taking exposures at different distances behind the primary shock front (determined by the $\mathrm{H} \alpha$ emission, Blair et al. 1999), we will examine the spatial variation of the O VI emission. We will also observe radiative shocks on the Eastern and Western limb of the Cygnus Loop. The optical morphology of the Eastern filament is very turbulent while the Western filament is more laminar. By comparing the shapes and centroids of the $\mathrm{O}$ VI and $\mathrm{C}$ III lines between filaments, we will study changes in the kinematics of the postshock flow (such as instability driven turbulence in the cooler gas).

\subsection{Crab Nebula}

The visible Crab Nebula is surrounded along much of its periphery by a thin [O III] "skin", visible in deep ground-based images. The skin is best understood as the cooling region behind a shock driven by the synchrotron nebula into an extended halo of freely expanding ejecta (Sankrit \& Hester 1997). We will observe a location at the edge of the visible remnant where strong $[\mathrm{Ne} \mathrm{V}]$ emission has been detected. Any O VI detection will be strong confirmation of the existence of shocked gas and will be a diagnostic for the shock velocity.

\subsection{Vela}

Most ultraviolet observations of SNRs have concentrated on optically bright filaments. However, in the Vela SNR, we will observe a region selected on the basis of its X-ray brightness which is faint in the optical. We expect to detect, in addition to O VI, emission lines from several species such as S VI, Si VII, Si VIII, Ne VII, Ar XII, and Ni XIV (some which have never before been detected in SNRs) and to get a more complete picture of the ionization structure of the gas.

Acknowledgments. This work is supported by NASA Contract NAS532985 to the Johns Hopkins University.

\section{References}

Blair, W. P., Sankrit, R., Raymond, J. C., \& Long, K. S. 1999, AJ, 118, 942

Levenson, N. A., Graham, J. R., Keller, L. D., \& Richter, M. J. 1998, ApJS, 118,541

Sankrit, R. \& Hester, J. J. 1997, ApJ, 491, 796 